\title{
Nucleic Acid Synthesis in Various Organs of Developing Human Fetuses
}

\author{
Anil B. Mukherjee ${ }^{[11]}$ and Claudia Hastings \\ Division of Human Genetics, Department of Pediatrics, State University of New York at Buffalo,
} Buffalo Children's Hospital, Buffalo, New York, USA

Maimon M. Cohen

Department of Human Genetics, Hadassah-Hebrew University Medical Center, Jerusalem, Israel

\begin{abstract}
Extract
In order to establish the mode of differentiation in various developing organs, the patterns of nucleic acid synthesis were studied in 14 human fetuses. The gestational age ranged from 8-15 weeks. Incorporation of radioactive precursors into DNA and RNA of cells in liver, kidney, heart, skin, spleen, lung, and brain were measured by autoradiography. Unique patterns of nucleic acid synthesis were observed in each of seven organs studied. The curves of DNA and RNA synthesis were "mirror images" of each other, which indicated organ-specific periodicity of nucleic acid synthesis.
\end{abstract}

\section{Speculation}

The findings of this study indicate periodicity and unique patterns of nucleic acid synthesis in developing organs of human fetuses at various gestational ages. It is possible that physical and chemical substances affecting nucleic acid synthesis of a particular organ may be most deleterious during this period.

\section{Introduction}

It has been suggested that differentiation may be regulated through the scheduled synthesis of the informational macromolecules (DNA and RNA) in those cells which constitute the primordial organs [1]. Although some studies have elucidated patterns of nucleic acid synthesis in developing organs of rat [8], mouse, and guinea pig [4], the only human organ thus far investigated has been the postnatal brain [7]. The present experiments were designed to study patterns of DNA and RNA synthesis in various organs of developing human fetuses at different gestational ages.

\section{Materials and Methods}

Fourteen human fetuses (each in an intact sac) were obtained immediately after hysterotomy performed for psychiatric reasons. The age of each embryo $(6,7,8$, $10,12,13,14$, and 15 weeks of pregnancy) was estimated from crown-rump measurements and menstrual histories. Two fetuses were examined at each gestational period, except for the 6 th and 15 th weeks, when only one specimen was available. Finely minced, small pieces of liver, kidney, heart, skin, spleen, lung; and brain were explanted into Ham's F-10 nutrient medium, supplemented with $20 \%$ fetal calf serum [9], 
which contained either ${ }^{3} \mathrm{H}$-uridine (sp act, 2,000 mCi/ $\mathrm{mm}$; final concentration, $2 \mu \mathrm{Ci} / \mathrm{ml}$ ) or ${ }^{3} \mathrm{H}$-thymidine (sp act, $1,900 \mathrm{mCi} / \mathrm{mm}$; final concentration, $2 \mu \mathrm{Ci} / \mathrm{ml}$ ). After incubation at $37^{\circ}$ in an atmosphere of $5 \% \mathrm{CO}_{2}$ in air for $1 \mathrm{hr}$, the tissues were washed several times in balanced salt solution and fixed in $45 \%$ acetic acid for $24 \mathrm{hr}$. Vigorous pipetting disrupted the cell clumps and cell suspensions were centrifuged at $800 \mathrm{rpm}$ and fixed in $3 / 1$ methanol-acetic acid for $30 \mathrm{~min}$. This was followed by several more changes of this fixative. The cell pellet was finally resuspended in $1.2 \mathrm{ml}$ fresh fixative. Cell suspension (2-3 drops) was placed on a microscope slide predipped in $70 \%$ methanol and quickly passed through a flame for "blaze" drying.

After extraction with cold 5\% trichloroacetic acid (TCA) (30 min) to remove unincorporated isotope, the slides were rinsed thoroughly in distilled water, dipped in Kodak nuclear track emulsion (type $N T B_{3}$ ), and kept at $4^{\circ}$ for 10 days. After developing, the slides were stained with buffered giemsa [6]. The percentage of labeled cells for each isotope was determined on the basis of 1,000 cells/organ/fetus. A cell with more than 20 grains was considered labeled. Because of TCA treatment of all slides before autoradiography, no background label was observed and therefore a background correction was not necessary. Treatment of test slides with DNAase and RNAase before coating with the emulsion resulted in no label, which indicated that the isotope was indeed incorporated directly into nucleic acids. The sex of each fetus (eight male and six female) was determined from chromosome analysis of cultured skin fibroblasts.

\section{Results}

Inasmuch as the difference in isotope incorporation between the two fetuses examined at any given gestational age was extremely small, average differences of ${ }^{3} \mathrm{H}$-uridine $=2.1 \% \pm 1.6 \%$ (range of $0.1-6.3 \%$ ) and ${ }^{3} \mathrm{H}$-thymidine $=2.3 \% \pm 1.6 \%$ (range $0.6-7.6 \%$ ), the observations were pooled and their mean value presented. At gestational periods when fetuses of both sexes were observed, no apparent sex difference in isotope uptake was observed in any organ. The incorporation patterns of ${ }^{3} \mathrm{H}$-uridine and ${ }^{3} \mathrm{H}$-thymidine into the various organs at different gestational times are shown in Figure 1.

\section{Liver}

${ }^{3} \mathrm{H}-$-Thymidine incorporation in the liver occurred at a relatively constant rate between 6 and 10 weeks of

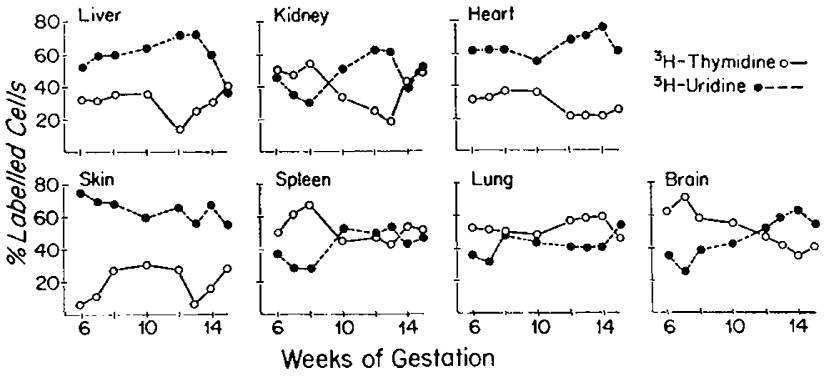

Fig. 1. The time course of DNA and RNA synthesis of various fetal organs at different gestational ages.

gestation. However, a sharp decrease in labeled cells was observed at 12 weeks, followed by a subsequent increase throughout the remainder of the periods studied. On the other hand, ${ }^{3} \mathrm{H}$-uridine incorporation, always at a higher level than ${ }^{3} \mathrm{H}$-thymidine, showed a gradual but steady increase, reaching a peak at week 13, followed by a rapid decline.

\section{Kidney}

Kidney cells showed a distinct periodicity of ${ }^{3} \mathrm{H}$-thymidine incorporation. The curve suggests a small peak at 8 weeks of gestation, then a marked decrease until week 13, followed by dramatic resumption of incorporation at weeks 14 and 15 . The ${ }^{3} \mathrm{H}$-uridine pattern of the kidney was almost an exact complement of the thymidine, in that the peak incorporation occurred at weeks 12 and 14 while the least label was observed in 8-week-old fetuses.

\section{Heart}

As in the liver, ${ }^{3} \mathrm{H}$-uridine incorporation was constantly higher than ${ }^{3} \mathrm{H}$-thymidine. Both isotopes showed little change in incorporation rates until week 10; then, ${ }^{3} \mathrm{H}$-uridine incorporation gradually increased while ${ }^{3} \mathrm{H}$-thymidine decreased until week 14 , when the trends were reversed.

\section{Skin}

The skin showed the widest discrepancy between the rates of incorporation of the two isotopes. The ${ }^{3} \mathrm{H}$-thymidine uptake was extremely low at week 6 but reached a peak at week 8 and maintained a plateau until week 12. At this time, a sharp decrease in incorporation for 1 week was followed by increased uptake at weeks 14 and 15 . This fluctuation in ${ }^{3} \mathrm{H}$-thymidine incorporation may be due to mixing of cell populations from different cell layers of the growing skin. 
The skin was perhaps the most active organ from the standpoint of ${ }^{3} \mathrm{H}$-uridine incorporation, because it had the highest percentage of labeled cells. The general trend of the curve indicated a gradual, but continuous, decline in ${ }^{3} \mathrm{H}$-uridine incorporation into the skin as gestation progressed.

\section{Spleen}

${ }^{3} \mathrm{H}$-Thymidine uptake increased rapidly from 6 to 8 weeks, folowed by a decline at week 10. ${ }^{3} \mathrm{H}$-Uridine incorporation demonstrated the reverse pattern. After the 10th week of gestation, relatively little change was evident in the rate of incorporation of either isotope.

\section{Lung}

The lung showed the least activity for any organ studied. Although an initial fluctuation in ${ }^{3} \mathrm{H}$-uridine uptake was apparent, from weeks $8-14$, the curves for both isotopes showed almost identical patterns. At week 15, however, a reversal of the trend appeared.

\section{Brain}

Brain cells showed the highest rate of ${ }^{3} \mathrm{H}$-thymidine incorporation during the earliest periods studied. Peak incorporation was observed at week 7 , followed by a gradual decrease until week if when a slight increase again appeared. ${ }^{3} \mathrm{H}$-Uridine uptake showed the reverse trend with the lowest incorporation occurring at week 7 , a gradual increase until week 14 , then a decline at 15 weeks.

\section{Discussion}

Because thymidine and uridine are integral components of DNA and RNA, the incorporation of these isotopes directly indicates nucleic acid synthesis in those cells investigated. Furthermore, since both RNA and DNA synthesis precede mitosis in all cells, except those undergoing polyploidy, the autoradiographic data obtained form a qualitative assessment of cell multiplication in various organs of the developing human fetus. Quantitative changes in DNA, RNA, and protein during prenatal and postnatal growth of the rat suggest that the earliest prenatal growth proceeds entirely by cell division [5]. Our data on human fetuses are in agreement with others $[3,7,8]$, which suggests that the rates of DNA and RNA synthesis have characteristic patterns in various organs. These patterns also suggest several waves of nucleic acid syn- thesis during different periods of growth of a given organ.

An obvious inverse relation between DNA and RNA synthesis exists since, in every organ studied, "mirror image" curves were obtained (Fig. 1). As the rate of DNA synthesis decreased, RNA synthesis increased and vice versa. However, at no point during the culture period did RNA synthesis completely cease.

Fish and Winnick [7] have demonstrated that postnatal development of the human brain occurs through an increase in cell number (mitosis) and size (maturation), and that both processes occur concurrently, but at different rates, yielding a definite periodicity. The apparent waves of DNA and RNA synthesis observed in different organs of the fetuses in our study could be interpreted as cell division followed by differentiation and/or metabolic activity [5]. The present investigations substantially support the observations of Fish and Winnick on human postnatal brain [7] and extend them to the development of additional organs during the prenatal period. These studies represent the first attempt in which patterns of nucleic acid synthesis and cellular activity have been examined for a group of human fetuses during such early gestational periods. Although descriptive and qualitative in nature, the data in the present investigation represent cellular activity during prenatal growth of human fetuses. These data establish the chronologic events in nucleic acid synthesis during normal growth and development of various organs and should provide a base line for further investigations of growth retardation in human fetuses and the effects of various drugs on these growth variables.

\section{Summary}

Unique patterns of DNA and RNA synthesis were observed in each of seven organs studied in eight male and six female human fetuses (gestational age, 6-15 weeks). The curve for DNA and RNA synthesis were "mirror images" of each other, which indicated an organ-specific periodicity of nucleic acid synthesis.

\section{References and Notes}

1. Davidson, E. H.: Gene Activity in Early Development, p. 1. (Academic Press, New York, 1968).

2. Enesco, M., and Leblond, C. P.: Increase in cell number as a factor in the growth of the organs of the young male rat. J. Embryol. Exp. Morphol. 10: 530 (1962).

3. Frsh, I., AND WrNick, M.: Cellular growth in various regions of the developing rat brain. Pediat. Res., 3: 407 (1969). 
4. Mandell, P., Rein, H., Harth-Edel, S., and Mardell, R.: Comparative Neurochemistry, p. 153. (Pergamon Press, London, 1964).

5. Pelc, S. R.: Turnover of DNA and function. Nature, 219: 162 (1968).

6. Schmid, W.: DNA replication patterns in human chromosomes. Cytogenetics, 2: 175 (1963).

7. WinNick, M.: Changes in nucleic acid and protein content of human brain during growth. Pediat. Res., 2: 352 (1968).

8. Winnick, M., AND Noble, A.: Quantitative changes in DNA, RNA and protein during prenatal and postnatal growth in rat. Develop. Biol., 12: 451 (1965).

9. Grand Island Biological Company, Grand Island, N.Y.
10. We would like to thank Dr. H. Robinson for supplying the human fetal material and Dr. R. Davidson for critical review of the manuscript.

11. This research was supported in part by Grants Department of Health, Education and Welfare, nos. HD-GM-06321 and HD 05187, National Institutes of Health, Lalor Foundation, and Project no. 417, Maternal and Child Health Branch.

12. Requests for reprints should be addressed to: ANIL B. MukherJeE, Ph.D., State University at New York at Buffalo, School of Medicine, Department of Pediatrics, 86 Hodge Ave., Buffalo, N.Y. 14222 (USA).

13. Accepted for publication March 12, 1973. 